\title{
SISTEMA DE GESTIÓN DE RESIDUOS SÓLIDOS HOSPITALARIOS PARA REDUCIR EL IMPACTO AMBIENTAL EN UN HOSPITAL DE SEGURIDAD SOCIAL DE TACNA - 2018.
}

\author{
HOSPITAL SOLID WASTE MANAGEMENT SYSTEM TO REDUCE THE ENVIRONMENTAL IMPACT \\ IN A HOSPITAL SOCIAL SEGURITY THE TACNA - 2018
}

\author{
Milagros Herrera Rejas ${ }^{1}$ \\ Richard Sabino Lazo Ramos²
}

Aceptado: $17 / 09 / 2019$

Publicado online:27/12/2019

\section{RESUMEN}

El objetivo del estudio se centró en elaborar una propuesta de Sistema de Gestión de residuos sólidos hospitalarios para reducir el impacto ambiental en el Hospital III Daniel Alcides Carrión de Tacna. El diseño de la investigación fue no experimental, transversal, prospectivo; por lo que se realizó un diagnostico basal a fin de llevar a cabo la caracterización de residuos sólidos hospitalarios en un periodo de siete días, y para la valoración de los componentes de gestión y manejo de residuos sólidos hospitalarios se aplicaron fichas de evaluación según se indica en la NTS $\mathrm{N}^{\circ}$ 096- MINSA/DIGESA, por lo que este procedimiento de evaluación permite identificar el impacto ambiental generado por el inadecuado manejo de residuos sólidos en todas sus etapas de manejo. Los resultados evidenciaron que; la clase de residuo de mayor generación corresponde a residuos Biocontaminados que alcanza valores promedio de $391 \mathrm{~kg} /$ día, seguido de residuos comunes que alcanza valores promedio de $112 \mathrm{~kg} /$ día y por último residuos especiales que representa $17 \mathrm{~kg} /$ día. En relación a la verificación de cumplimiento de aspectos administrativos y de gestión de manejo de residuos sólidos en el Hospital, siguiendo la aplicación de la norma vigente NTS $N^{\circ}$ 096- MINSA/DIGESA se determina que primero: para las Etapas de manejo de residuos sólidos ha obtenido una valoración de aceptable; segundo para cumplimiento de aspectos de Gestión de

\footnotetext{
${ }^{1}$ Maestro en Gestión Ambiental y Desarrollo Sostenible, Docente de la Facultad de Ingeniería de la Universidad Privada de Tacna, Tacna, Perú

2 Doctor en Ciencias Ambientales, Director de la Escuela Profesional de Ingeniería Ambiental de la Universidad Privada de Tacna, Tacna, Perú
} 
residuos, ha obtenido una calificación aceptable donde ha obtenido un puntaje de 30. Por tanto, estos resultados han permitido elaborar una propuesta de diseño de sistema de manejo de residuos sólidos hospitalarios para reducir el impacto ambiental en el Hospital III Daniel Alcides Carrión documento que permitirá fortalecer y mejorar el manejo adecuado de los residuos y así contribuir a la prevención y control de la contaminación sanitaria y ambiental.

Palabras clave: contaminación ambiental, residuos hospitalarios, hospital.

\section{ABSTRACT}

The objective of this study was to elaborate a proposal of a hospital waste management System to reduce the environmental impact in the Hospital III Daniel Alcides Carrión of Tacna. The design of this research was non experimental, transversal and prospective because of the realization of a basal diagnostic to the characterization of the hospital waste in a period of seven days, and to valorize the components of hospital waste management were applicate evaluation cards as it is indicated in the Technical Health Standard of Peru NTS $N^{\circ}$ 096MINSA/DIGESA this procedure of evaluation permit to identify the environmental impact generated because of the inappropriate waste management in all their stages. The results showed that the type of waste which is generated the most is biocontaminated with 391 $\mathrm{kg} /$ day followed of the common waste with $112 \mathrm{~kg} / \mathrm{day}$ and at last the special waste with $17 \mathrm{~kg} /$ day. Related to the compliance of the administrative aspects and waste management in the hospital as the NTS $N^{\circ}$ 096- MINSA/DIGESA said: for the waste management stages it obtained a valuation of good, secondly for the compliance of waste management aspects it obtained a valuation of good with a punctuation of 30 . There for this results have permitted to elaborate a proposal of hospital waste management system to reduce the environmental impact at the Hospital III Daniel Alcides Carrión of Tacna, document that will allow to strengthen and improve the suitable hospital waste management and contributes to the prevention and control of the sanitary and environmental pollution.

Key words: environmental pollution, hospital waste, hospital.

\section{INTRODUCCCIÓN}

Actualmente en un contexto normativo ambiental se ha venido actualizando las diferentes normas ambientales, en ese contexto tenemos una nueva Ley de Gestión Integral de Residuos Sólidos, documento que hace énfasis en el uso eficiente de los materiales y asegurar una gestión y manejo de los residuos sólidos que comprende la 
minimización de la generación de los residuos sólidos en la fuente, la valorización, tratamiento y disposición final sanitaria y ambientalmente segura; bajo estos alcances los residuos que se generan en los diferentes servicios de atención hospitalaria corresponden a residuos peligrosos por lo que se hace necesario conocer las características de peligrosidad de estos residuos. Así mismo estos residuos representan un riesgo significativo para la salud o el ambiente; donde muchas veces ha restado importancia entre los actores involucrados para la solución de esta problemática derivada de un deficiente manejo de residuos hospitalarios producto de una atención hospitalaria. Para tal efecto el desarrollo del presente trabajo de investigación pretende elaborar un Sistema de Gestión de Residuos Sólidos Hospitalarios para reducir el impacto ambiental en el Hospital III Daniel Alcides Carrión el mismo que ha sido desarrollado con un enfoque técnico, participativo y de comunicación con todos los actores involucrados a fin de implementar los diferentes alcances que dicta la normativa legal en el diseño del presente sistema de gestión.

Actualmente, el manejo de residuos hospitalarios en dicho establecimeinto requiere mejoras. Los residuos son transportados a un área de almacenamiento temporal para luego ser entregados a una EO - RS (Empresa Operadora de Residuos Sólidos) para su transporte externo y disposición final a un relleno de seguridad autorizado por el sector competente; sin embargo debido a las condiciones contractuales con esta empresa y al limitado presupuesto asignado a la administración del Hospital; estos procesos representan un alto riesgo sanitario y ambiental por el inadecuado manejo y almacenamiento de los residuos peligrosos al permanecer por más de 48 horas en el almacén temporal. Se estima una generación diaria de residuos peligrosos de 350 a 450 $\mathrm{kg} /$ día, valores que podrían reducirse y minimizar su volumen de generación, si el personal asistencial realiza de manera efectiva la práctica de segregación en Fuente. Actualmente en la Ciudad de Tacna no existe relleno de seguridad, para disposición de residuos peligrosos. Desde un aspecto social, existen problemas técnicos de segregación en la fuente de origen de residuos según su clase, lo que debe estar relacionado a la deficiente capacitación ó actitud para realizar una adecuada segregación en la fuente, lo que ocasiona incremento de peso y volumen de estos residuos; y pone en cuestión no realizar prácticas de minimización de residuos y la valorización de estos. Quijano (2017), indicó que la cultura organizacional es importante en el manejo de residuos sólidos hospitalarios. Yactayo (2013), en el Hospital Nacional Dos de mayo de Lima indica, que la actual gestión y manejo de residuos es inadecuada por la carencia de un Modelo de Gestión. El Ministerio del Ambiente (2008) en el informe de la Situación actual de la Gestión de Residuos no Municipales indica que el sector salud, no cuenta con información sistematizada en DIGESA en referencia a las provincias y que el sector genero un total de 1143,21 TM de residuos peligrosos, no se diferencian los residuos biocontaminados de los especiales. Canahuire S. (2013) en su trabajo de investigación de los residuos sólidos generados en el Hospital Regional Hipólito Unanue determina que el $66 \%$ en peso de residuos son biocontaminados y el $34 \%$ son residuos comunes; y el servicio que genera mayor cantidad de residuos es el servicio de medicina que es $25.92 \mathrm{Kg} /$ día, seguido del servicio de ginecología con $23.49 \mathrm{~kg} /$ día de residuos. 
Un sistema de gestión ambiental (SGA) se define como un sistema estructurado de gestión, integrado en la actividad de gestión de la organización (Prieto González, María. 2011). (Ley de Gestión Integral de Residuos Sólidos, D.L 1278 Definición de términos).

\section{OBJETIVOS}

El objetivo fue determinar la composición, características, cantidad, de los residuos sólidos generados en el Hospital III Daniel Alcides Carrión de Tacna, 2018, y proponer una gestión eficiente de los residuos sólidos generados en el Hospital III Daniel Alcides Carrión de Tacna 2018, para reducir el impacto ambiental.

\section{METODOLOGÍA}

Investigación, no experimental, observacional de corte transversal. El estudio se realizó en el Hospital III Tacna "DANIEL ALCIDES CARRIÒN" de la Red asistencial de Es Salud, Centro asistencial categorizado con Resolución Directoral Na 146-2014- de OAJ-DESPDR/DRST/GOB.REG.TACNA, como Hospital Categoría III-3, con código Registro SUNASA $N^{\circ}$ 1304566. Así mismo, el Hospital Base de la Red Asistencial de Tacna no cuenta con población adscrita al constituirse un hospital referencial de los Centros de Atención Primaria de la Red de Es Salud. El tamaño de la muestra fue de 23 servicios de atención hospitalarias y 461 personas que realizan labores asistenciales. La metodología aplicada se basó en el método de observación, diagnostico y aplicación de listas de cotejo de evaluación de las condiciones sanitaria y ambiental en la adecuada gestión de manejo de los residuos sólidos hospitalarios en el servicio médico de apoyo, en cumplimiento de normativa legal. Para la fase de diagnóstico, caracterización y manejo de residuos sólidos se aplicó los términos de referencia que se indican en NTS N. ${ }^{\circ} 096-$ MINSA/DIGESA V.01.

\section{RESULTADOS}

Los residuos hospitalarios que se generan dentro del ámbito de los servicios del Hospital producto de las atenciones medicas son de riesgo ambiental y sanitario su manejo; para lo cual se ha desarrollo la fase de diagnóstico y caracterización desarrollados en siete días lo que permitirá identificar el impacto ambiental que se produce por el inadecuado manejo; inicialmente se realizó muestreos previos de generación de residuos observándose que las resultados son poco significativos, llegándose a optimizar la muestra.

En table 1 se muestra la identificación de residuos generados en el hospital, según áreas críticas de atención hospitalaria y por clase de residuo: biocontaminados, especiales y comunes. El total de residuos generados en siete días alcanza un valor promedio de $391 \mathrm{~kg} /$ día, seguido de los residuos comunes que alcanza valor promedio diario de 112 $\mathrm{kg} /$ día y por ultimo residuos especiales que alcanza valores promedio de $17 \mathrm{~kg} /$ día. Un $75 \%$ corresponde a residuos biocontaminados, seguido de un $22 \%$ de residuos comunes y un $3 \%$ de residuos especiales, del total de residuos generados durante el desarrollo del diagnóstico de siete días decaracterización. (Figura 1) 
Tabla 1

Identificación de características de peligrosidad por áreas críticas

\begin{tabular}{|c|c|c|c|}
\hline \multirow[b]{2}{*}{ ÁREAS CRÍTICAS } & \multicolumn{3}{|c|}{ TIPO DE RESIDUO } \\
\hline & $\begin{array}{l}\text { RESIDUOS CLASE A: } \\
\text { BIOCONTAMINADOS }\end{array}$ & $\begin{array}{l}\text { RESIDUOS CLASE B: } \\
\text { ESPECIALES }\end{array}$ & RESIDUOS CLASE C \\
\hline HOSPITALIZACIÒN & $\begin{array}{l}\text { A.1 Atención al paciente } \\
\text { A.3 Bolsas de sangre } \\
\text { A.5 Elementos } \\
\text { Punzocortantes }\end{array}$ & $\begin{array}{l}\text { B.2 Residuos } \\
\text { Farmacéuticos }\end{array}$ & $\begin{array}{l}\text { Papeles, envolturas de } \\
\text { guantes, envases } \\
\text { descartables, cartones, } \\
\text { etc }\end{array}$ \\
\hline $\mathrm{UCl}$ & $\begin{array}{l}\text { A.1 Atención al paciente } \\
\text { A. } 3 \text { Bolsas de sangre } \\
\text { A.5 Elementos } \\
\text { Punzocortantes }\end{array}$ & $\begin{array}{l}\text { B.1 Residuos químicos } \\
\text { peligrosos } \\
\text { B.2 Residuos } \\
\text { Farmacéuticos }\end{array}$ & $\begin{array}{l}\text { Papeles, envolturas de } \\
\text { guantes, envases } \\
\text { descartables, cartones, } \\
\text { etc }\end{array}$ \\
\hline EMERGENCIA & $\begin{array}{l}\text { A.1 Atención al paciente } \\
\text { A.3 Bolsas de sangre } \\
\text { A.4 Residuos quirúrgicos y } \\
\text { patológicos } \\
\text { A.5 Elementos } \\
\text { Punzocortantes }\end{array}$ & $\begin{array}{l}\text { B.1 Residuos químicos } \\
\text { peligrosos } \\
\text { B.2 Residuos } \\
\text { Farmacéuticos }\end{array}$ & $\begin{array}{l}\text { Papeles, envolturas de } \\
\text { guantes, envases } \\
\text { descartables, cartones, } \\
\text { etc }\end{array}$ \\
\hline LABORATORIO & $\begin{array}{l}\text { A.1 Atenció+C3n al paciente } \\
\text { ! Biológicos } \\
\text { B Bolsas de sangre } \\
\text { A.4 Residuos quirúrgicos y } \\
\text { patológicos } \\
\text { A.5 Elementos } \\
\text { Punzocortantes }\end{array}$ & $\begin{array}{l}\text { B.1 Residuos químicos } \\
\text { peligrosos } \\
\text { B.2. Residuos } \\
\text { farmacéuticos } \\
\text { B.3 Residuos radiactivos }\end{array}$ & $\begin{array}{l}\text { Papeles, envolturas de } \\
\text { guantes, envases } \\
\text { descartables, etc }\end{array}$ \\
\hline QUIMIOTERAPIA & $\begin{array}{l}\text { A.1 Atención al paciente } \\
\text { A.5 Elementos } \\
\text { Punzocortantes }\end{array}$ & $\begin{array}{l}\text { B.2 Residuos químicos } \\
\text { peligrosos } \\
\text { B.3 Residuos radiactivos }\end{array}$ & $\begin{array}{l}\text { Papeles, envolturas de } \\
\text { guantes, envases } \\
\text { descartables, cartones, } \\
\text { etc }\end{array}$ \\
\hline TÓPICO & $\begin{array}{l}\text { A.1 Atención al paciente } \\
\text { A.5 Elementos } \\
\text { Punzocortantes }\end{array}$ & $\begin{array}{l}\text { B.2 Residuos } \\
\text { Farmacéuticos }\end{array}$ & $\begin{array}{l}\text { Papeles, envolturas de } \\
\text { guantes, envases } \\
\text { descartables, cartones, } \\
\text { etc }\end{array}$ \\
\hline $\begin{array}{l}\text { CONSULTORIO } \\
\text { EXTERNO }\end{array}$ & $\begin{array}{l}\text { A.1 Atención al paciente } \\
\text { A.5 Elementos } \\
\text { Punzocortantes }\end{array}$ & $\begin{array}{l}\text { B.2 Residuos } \\
\text { Farmacéuticos }\end{array}$ & $\begin{array}{l}\text { Papeles, envolturas de } \\
\text { guantes, envases } \\
\text { descartables, cartones, } \\
\text { etc }\end{array}$ \\
\hline
\end{tabular}

Porcentaje promedio en peso por tipo de residuo, del 8 al 14 de julio del 2018

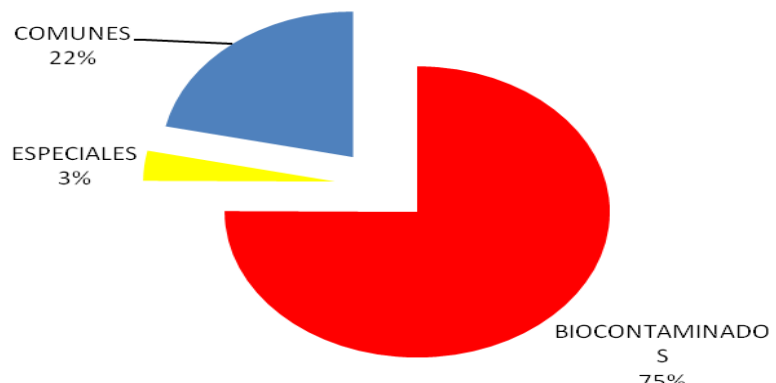

$75 \%$

Figura 1

Porcentaje promedio en peso por clase de residuo, del 8 al 14 de julio de 2018 
La generación de residuos biocontaminados en siete días de muestreo por servicio de salud, muestran una generación diaria promedio de $366 \mathrm{~kg} /$ día; del mismo modo los resultados obtenidos muestran que el servicio de mayor generación de residuos biocontaminados es Hospitalización medicina que presenta un mayor índice de 76 $\mathrm{kg} /$ dia, seguido del servicio de hemodiálisis con $54 \mathrm{~kg} /$ dia y el servicio de Nutrición y dietética con un índice $41 \mathrm{~kg} /$ dia respectivamente. En la figura 2 se observa que los servicios que generan mayor cantidad de residuos biocontaminados son el servicio de Hospitalización y medicina que genera $535 \mathrm{~kg} / \mathrm{semana}$ aproximadamente, seguido del servicio de Hemodiálisis que genera $380 \mathrm{~kg} / \mathrm{semana}$, el servicio de Nutrición y dietética $290 \mathrm{~kg} / \mathrm{semana}$.

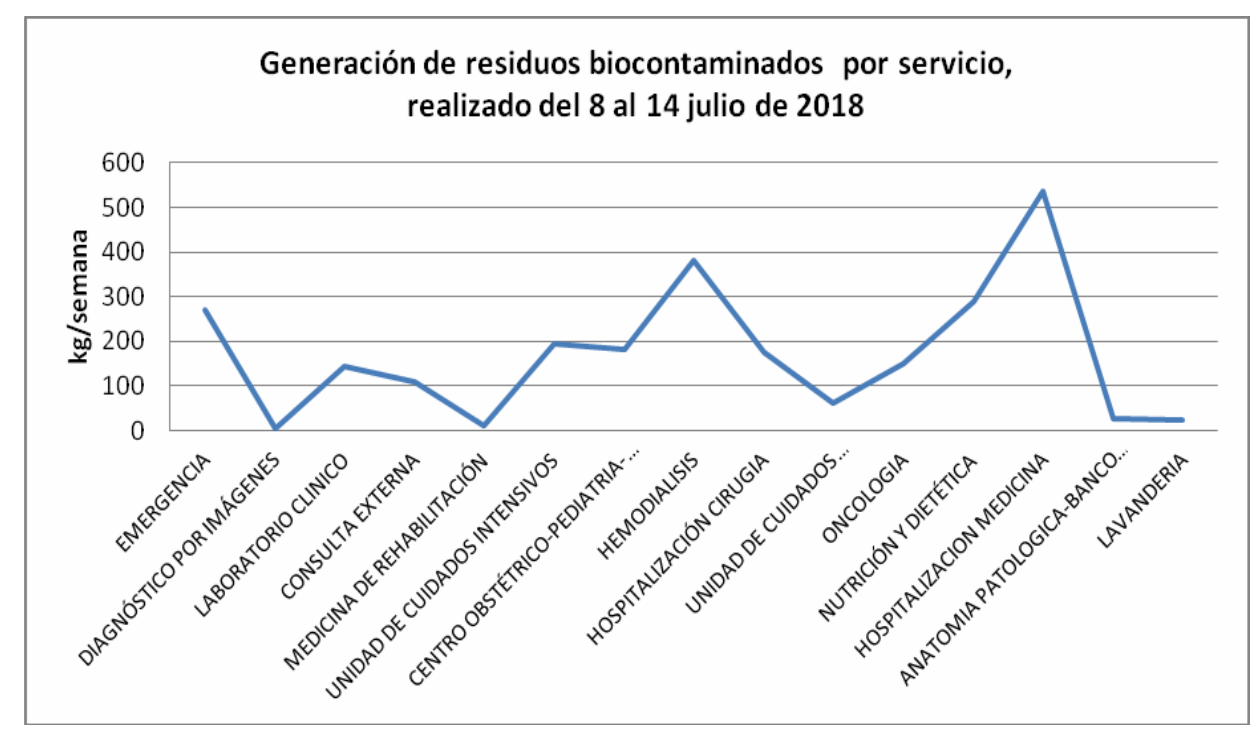

Figura 2

Generación de residuos biocontaminados por servicio

El Hospital cuenta con recipientes adecuados para el almacenamiento de residuos en la fuente de generación primaria. Se realizó el recorrido al interior del hospital, verificándose la señalización de rutas de transporte de residuos sólidos en el hospital; no observándose un mapa de ruta de transporte de residuos hospitalarios. Para realizar la verificación del manejo de residuos sólidos, respect acondicionamiento, los servicios de hospitalización, emergencia, pediatría, entre otros se observó que algunos recipientes para residuos biocontaminados, comunes y especiales, no cuentan con su respectiva tapa. En el servicio de Hospitalización cirugía se observó que los recipientes de residuos punzocortantes (galoneras) no tenían rotulo ni símbolo de bioseguridad. El servicio de laboratorio no cuenta con recipientes para residuos especiales. En el servicio de Tópico de enfermería se observó recipiente para residuos biocontaminados no tienen tapas, los recipientes de punzocortantes (galoneras) no tenían rotulo ni símbolo de bioseguridad. Algunos servicios se ha observado bolsas de color rojo, para almacenar residuos biocontaminados y estos se encuentran colgados en una mesita de atención hospitalaria. Respecto a segregación y almacenamiento primario, se identificó áreas críticas de atención medica como emergencia, hospitalización, tópico, entre otros, donde se evidencio hallazgos de no conformidad dado que se observo inadecuadas 
prácticas de segregación al disponer residuos comunes (empaques de aguja, de guantes, de gasas) al interior del recipiente para residuos biocontaminados. En esta etapa de recolecciòn y transporte interno, se observa que la recolección y transporte de residuos al área de almacenamiento intermedio se realiza con frecuencia diaria y por turno, además de observándose que cuenta con contenedores con ruedas. Se cuenta con 7 áreas de almacenamiento intermedio, los mismos que se encuentran medianamente acondicionados. El área de almacenamiento central se encuentra ubicado en la parte posterior del hospital, evidenciándose que los residuos biocontaminados son apilados en esta área por más de siete a quince días; acción que se encuentra supeditada al servicio de recojo de la empresa operadora de residuos sólidos. (Figura 3)

\section{Figura 3}

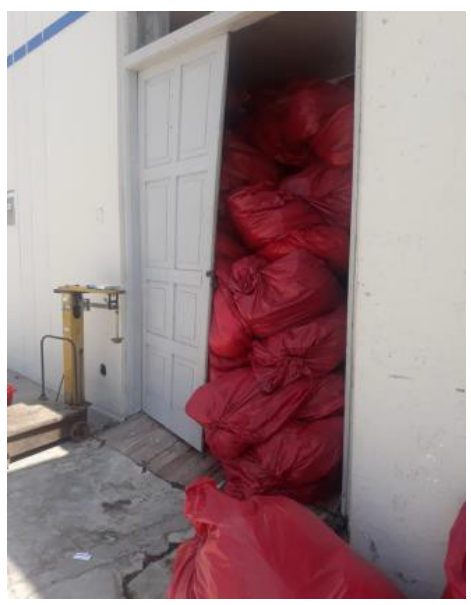

Vista fotográfica de área de almacenamiento final

El Hospital cuenta con Equipo de tratamiento de residuos sólidos peligrosos de tipo incinerador, el mismo que se encuentra inoperativo. Del mismo modo indicar que este equipo no cuenta con la Certificación Ambiental otorgado por el sector competente. Se realizó la verificación de cumplimiento de aspectos administrativos y de gestión para lo cual se aplico la Ficha N 01: Verificación de Cumplimiento de los Aspectos de Gestión de Residuos Sólidos en Establecimientos de Salud y Servicios Médicos de Apoyo, de la DIGESA-MINSA; obteniéndose los siguientes resultados:

El Hospital III Daniel Alcides Carrión cuenta con un Comité de Gestión y Manejo de Residuos Sólidos, para lo cual cuenta con documento aprobado por Resolución de Gerencia de Hospital. No se evidenció en la información revisada el Reglamento del comité; pero este documento se encuentra en proceso de formulación y además de contar con Libro de actas de reuniones. No se evidenció documentos de la realización de diagnóstico inicial.

De la evaluación y aplicación de criterios de valoración: Verificación de Cumplimiento de los Aspectos de Gestión de Residuos Sólidos en Establecimientos de Salud y Servicios Médicos de Apoyo, de la DIGESA-MINSA, se determina que ha obtenido una calificación de ACEPTABLE, para lo cual ha obtenido un puntaje de 36 . 


\begin{tabular}{|l|l|l|l|}
\hline \multicolumn{4}{|c|}{ CRITERIOS DE VALORACIÓN } \\
\hline MUY DEFICIENTE & DEFICIENTE & ACEPTABLE & SATISFACTORIO \\
\hline Puntaje menor o igual a 10 & Puntaje entre 11 y 21 & Puntaje entre 22 y 40 & Puntaje de 41 \\
\hline Fuente: DIGESA/ MINSA & & & \\
\hline
\end{tabular}

\section{DISCUSIÓN}

En referencia a la fase de diagnóstico concordamos con lo que concluye el Plan de Gestión Integral de Residuos Hospitalarios y Similares realizado por la Universidad de Cauca Colombia ya que la aplicación de listas de chequeo, en nuestro caso listas y fichas de verificación, permiten obtener el diagnóstico de la situación actual de la gestión de los residuos sólidos hospitalarios en el Hospital Daniel Alcides Carrión de Tacna a partir del cual se identifican las oportunidades de mejora y permiten proponer un sistema de gestión de residuos sólidos. De acuerdo a la verificación de los aspectos administrativos de la gestión de los residuos realizada en el presente estudio para el Hospital III Daniel Alcides Carrión de Tacna se obtuvo una evaluación ACEPTABLE la cual difiere del estudio realizado por Quijano Anacleto, Sirena (2017) Diagnóstico del manejo de residuos sólidos hospitalarios en el Hospital de Apoyo I "Santiago Apóstol" - Lambayeque la cual obtuvo una calificación de MUY DEFICIENTE, sin embargo cabe mencionar que ambos estudios coinciden en que es importante contar con un Modelo de Gestión más allá de sólo la aplicación y cumplimiento de la Norma Técnica de Salud № 096 - MINSA/DIGESA. En el presente estudio se muestra que si bien existen deficiencias en el almacenamiento final de los residuos hospitalarios, estos son dispuestos a través de una EO-RS en un relleno de seguridad, a diferencia de lo que sucede en los centros de salud del MINSA que de acuerdo al estudio "Evaluación del manejo de los residuos sólidos hospitalarios en el Centro de Salud de Ciudad Nueva del distrito de Ciudad Nueva-Tacna" realizado por Quispe C. (2015) estos residuos son dispuestos en el botadero municipal de Tacna, lo cual deja en evidencia que la problemática de los residuos hospitalarios es aún más álgida en el sector salud. Respecto a las cantidades de residuos hospitalarios generados en el presente estudio se muestran que los residuos biocontaminados representan el mayor porcentaje del total de residuos generados así mismo el servicio de mayor generación de residuos biocontaminados es el de hospitalización medicina, lo cual concuerda con lo mencionado en el estudio "Caracterización de los residuos sólidos generados en el Hospital Regional Hipólito Unanue en la Provincia de Tacna, 2013" realizado por Canahuire S. Del mismo modo, según el Plan Nacional de Gestión Integral de Residuos sólidos 2016 a 2024, se estima que para el sector salud de 548 establecimientos genera un total de 12755 ton/año, no precisando informes de hospitales de Es Salud y para el periodo 2008 según el Informe anual del Ministerio del Ambiente presenta resultados de generación de residuos hospitalarios del Policlínico Pablo Bermúdez donde produce 2,09 ton/año de residuos hospitalarios; por tanto el desarrollo del presente estudio ha permitido conocer con detalle la clase de residuos, peso, volumen y disposición final que se generan en el Hospital Daniel Alcides Carrión de Es Salud de Tacna. Se entregó una propuesta de Gestión al finalizer la investigación. 
La organización deberá tener presente la importancia de realizar esfuerzos para el cumplimiento de los compromisos ambientales y legales, y que está enfocado en procesos de mejora continua. La mejora continua implica desde la implementación del sistema, aprendizaje continuo a través del fortalecimiento de capacidades en todos los niveles de la organización y la participación activa de todos los actores involucrados; desde la alta gerencia y todos los trabajadores.

Se recomienda a las entidades competentes promover la creación de las condiciones que permitan a todos los establecimientos de salud cumplir con la normativa referente a la gestión de los residuos sólidos hospitalarios como, por ejemplo, promover ferias de exposición de tecnologías para el tratamiento de residuos sólidos hospitalarios, así mismo invitar a empresarios que puedan invertir en plantas para el tratamiento y/o disposición final de residuos hospitalarios. Realizar programas constantes de capacitación, sensibilización y educación a la población involucrada en el Hospital Daniel Alcides Carrión de ESSALUD Tacna respecto al manejo y disposición adecuada de los residuos sólidos hospitalarios para reducir la generación de los mismos.

Respecto a la disposición final de los residuos sólidos hospitalarios, se recomienda a ESSALUD poner en funcionamiento su incinerador pirolítico y gestionar su respectiva certificación ambiental ya que según lo evaluado el costo del tratamiento mediante esta técnica está entre 0.76 a 1.02 soles por kilogramo de residuo, de tal manera se evitaría la problemática con la EO-RS respecto al tiempo de atención en la recolección de los residuos y la sobre acumulación en el almacén temporal de residuos. Al comité de residuos sólidos del Hospital III Daniel Alcides Carrión de Tacna realizar supervisiones mensuales inopinadas a los diferentes servicios con la finalidad de evaluar las condiciones de segregación, almacenamiento temporal y final de los residuos hospitalarios. Convocar a todos los jefes de servicio sobre todo a aquellos de mayor generación de residuos con la finalidad de exponer los resultados de las supervisiones realizadas respecto al manejo de los residuos sólidos en sus respectivos servicios con la finalidad de comprometerlos a la identificación de las causas de las acciones reportadas así como a proponer y ejecutar las medidas correctivas correspondientes las cuales deberán quedar plasmadas en un acta y verificar su cumplimiento en la sucesiva reunión.

\section{REFERENCIAS BIBLIOGRÁFICAS}

Alegre (2007). Guía para el manejo de residuos sólidos enciudades

Batista P., Hernandez R., Fernandez C., (1998) Metodología de la investigación. México.

Canahuiri S. (2016) Caracterización de los residuos sólidos generados en el Hospital Regional Hipólito Unanue en la Provincia de Tacna, Perú.

Centro Panamericano De Ingeniería Sanitaria Y Ciencias Del Ambiente. (CEPIS). 1994. Guía para el manejo interno de residuos sólidos en centros de atención de salud. Perú. 49 p.

Cointreau-Levine, S (1998). Occupational and Environmental Health Issues of Solid Waste Management. Estados Unidos de América.

Condori C. (2017). Análisis y diagnóstico de la producción y gestión de residuos sólidos urbanos en el ámbito de influencia del espacio natural Doñana. Sevilla. España. 
DIGESA. (2010). Plan Nacional de Gestión de Residuos Sólidos en establecimientos de salud y servicios médicos de Apoyo 2010-2012; DIGESA-MINSA-PERU, 2010

Erazo, M (2007) Plan de manejo de residuos hospitalarios. estudio de caso: complejo asistencial Dr. Sótero Del río. Santiago.Chile.

Luis F., Marmolejo R, Carlos A., Gestión de los residuos sólidos en hospitales locales del norte del Valle del Cauca, Colombia. (2010)

MINISTERIO DE SALUD. (1995). Diagnóstico situacional del manejo de los residuos sólidos de hospitales administrados por el Ministerio de Salud. Lima.

MINISTERIO DE SALUD - Buenos Aires - Guía de capacitación para la gestión de residuos en establecimientos de atención de salud. 2017

MINISTERIO DE SALUD. (2010-2012). Plan Nacional de Gestión de Residuos Sólidos en Establecimientos de Salud y Servicios Médicos de Apoyo. Lima, Perú.

MINISTERIO DE SALUD - Guía técnica de procedimientos de limpieza y desinfección de ambientes en los establecimientos de salud y servicios médicos de apoyo. Resolución Ministerial 372-2011/MINSA

MINISTERIO DE SALUD - DIRECCION GENERAL DE SALUD AMBIENTAL E INOCUIDAD ALIMENTARIA (2011). Manual de Implementación del programa de prevención de accidentes con materiales punzocortantes en servicios de salud

MINISTERIO DE SALUD - MINISTERIO DEL AMBIENTE (2002). Manual de Procedimientos para la Gestión Integral de Residuos Hospitalarios y similares en Colombia. Colombia.

Navarro Y Gutiérrez (2007) Mejoramiento de la gestión integral de residuos sólidos municipales en el distrito de Tacna y la disposición final de residuos sólidos municipales en la ciudad de Tacna, provincia de Tacna, departamento de Tacna.

ORGANIZACIÓN MUNDIAL DE LA SALUD (OMS) 2007. Residuos de establecimientos de salud.

Plan Nacional de Gestión Integral de Residuos Solidos 2016-2024, Ministerio del Ambiente (2007).

Pruss A, Giroult E. y Rushbrook P., Manejo seguro de residuos establecimientos de salud, Organización Mundial de la Salud. (1999).

Quispe C. (2015) Evaluación del manejo de residuos sólidos hospitalarios en el Centro de Salud Ciudad Nueva del distrito de Ciudad Nueva -Tacna"

Quijano M. (2016) Diagnóstico del manejo de residuos sólidos hospitalarios en el Hospital de Apoyo I Santiago Apóstol Utcubamba.

Resolución № 554-2012/MINSA, se aprueba la NTS N096- MINSA/DIGESA V.01. Norma técnica de salud de gestión y manejo de residuos sólidos en establecimientos de salud y servicios médicos de apoyo. Perú.

Ruthala, William ET AL. (1992) Infection Control and Hospital Epidemiology.

Tejada P. Y Matos M. (2010) Manejo de residuos sólidos en la ciudad de Tacna revista Ciencia y desarrollo UNJBG.

Tello, P. (1991) Diagnóstico Situacional de los Residuos Sólidos de Hospitales en la Ciudad de Lima Metropolitana., Lima, Perú.

Vásquez, A. (2010). Programa de adecuación y manejo ambiental del incinerador pirolítico del Hospital Almanzor Aguinaga Asenjo -Chiclayo. EsSalud, Región Lambayeque.

Zabala M., Manual para el Manejo de Desechos en Establecimientos de Salud - Centro Panamericano de Ingeniería Sanitaria (1999) 